\title{
Dural venous sinus thrombosis: A rare complication of pulse corticosteroid therapy in pemphigus vulgaris
}

\author{
Vijay Gandhi, Sonia Agrawal, Sheetal Yadav, Sushobhan Saha
}

\author{
Department of Dermatology \& STD, UCMS \& GTB Hospital, Delhi, 110095, India \\ Corresponding author: Sonia Agrawal, MD, E-mail: Sonia.03agrawal@gmail.com
}

\begin{abstract}
Systemic pulse corticosteroid therapy is used widely in the treatment of pemphigus vulgaris. Dural venous sinus thrombosis as a complication of pulse therapy has not been reported in the literature. A middle-aged female with pemphigus vulgaris was started on monthly pulse dexamethasone therapy with daily azathioprine as an adjuvant. After two pulse therapies, she developed throbbing headache, which on further evaluation was determined to be due to dural venous thrombosis. Other causes of dural venous thrombosis were excluded. Pulse therapy was stopped and other medications were started. The headache subsided within two weeks of stopping pulse therapy. Corticosteroids may play the role of a procoagulant in producing cerebral venous sinus thrombosis. Herein, we report a rare case of dural venous sinus thrombosis due to pulse steroid therapy in pemphigus vulgaris.
\end{abstract}

Key words: Venous sinus thrombosis; pulse therapy; pemphigus

\section{INTRODUCTION}

Pemphigus vulgaris is a rare autoimmune bullous dermatosis that presents with intraepidermal blisters affecting the skin and mucosae. It results from the formation of $\operatorname{IgG}$ autoantibodies against the transmembrane desmosomal glycoproteins DSG3 and DSG1, leading to the loss of cell-to-cell adhesion (acantholysis) [1]. Therapeutic options include systemic corticosteroids, immunosuppressives such as azathioprine and cyclophosphamide, and more recently rituximab, which is a chimeric monoclonal antibody to CD20 present on B cells and pre-B cells [2]. Systemic steroids remain the mainstay of treatment. Systemic steroids are administered conventionally in daily doses. Steroids may also be administered as pulse therapy, in which high doses of methylprednisolone or dexamethasone are given intravenously for three days every four weeks. It is proposed that pulse therapy, apart from the induction of a quicker remission, also results in less HPA suppression than conventional daily doses. However, such high doses also involve some inherent risks, including volume overload, electrolyte disturbances, and cardiac rhythm disturbances, mainly bradyarrhythmias. The efficacy of dexamethasonecyclophosphamide pulse therapy in patients with pemphigus was documented by Pasricha et al. in an Indian setting [3]. Since the advent of steroid pulse therapy, there has been a radical improvement in the survival of these patients [4]. We report a case of dural venous sinus thrombosis in a patient with pemphigus vulgaris on corticosteroid pulse therapy, which, to the best of our knowledge, has not been described in the literature.

\section{CASE REPORT}

A 40-year-old female—a resident of New Delhi, Indiapresented to the outpatient dermatology department complaining of multiple fluid-filled lesions on the chest, back, and upper limbs. A clinical examination revealed multiple flaccid vesicles and bullae ranging in size from $0.5 \times 0.5 \mathrm{~cm}^{2}$ to $2 \times 2 \mathrm{~cm}^{2}$ on the chest, upper back, arms, and forearm. The lesions were filled with clear fluid and lay on non-erythematous bases. The bulla-spread sign and Nikolsky's sign were positive.

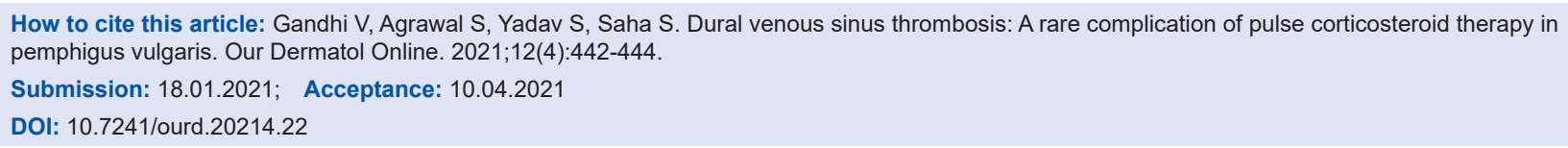


A presumptive diagnosis of pemphigus vulgaris was reached and the patient was investigated. A Tzanck smear from the vesicle revealed acantholytic cells. An H\&E-stained skin biopsy revealed a suprabasal split in the epidermis with acantholytic cells in the blister cavity. On direct immunofluorescence, IgG and C3 deposits in a fishnet pattern were observed on cell surfaces. An enzyme-linked immunosorbent assay (ELISA) determined that anti-nuclear antibody (ANA) was negative. Based on the clinical examination and laboratory parameters, the diagnosis of pemphigus vulgaris was thus made.

The patient was started on dexamethasone pulse therapy every four weeks with azathioprine $50 \mathrm{mg}$ twice daily as an adjuvant. Immediately after the second pulse therapy, the patient developed throbbing headache, which did not subside with analgesics. The vitals, including blood pressure, ECG, serum electrolytes, and a thyroid profile were normal. A fundus examination performed to exclude benign intracranial hypertension revealed small hyperemic discs, shallow cup margins with normal vessels. The intraocular tension on tonometry was normal. A neurologist's opinion was taken and MRI angiography of the brain was advised. MRI of the brain showed a lack of normal flow voids in the right transverse and sigmoid venous dural sinuses, which was suggestive of dural venous thrombosis. Magnetic resonance venography (MRV) revealed the attenuation of flow-related enhancement of the right transverse sinus and the most distal part of the superior sagittal sinus with non-visualization of the right sigmoid sinus (Fig. 1).

Investigations were done to evaluate the cause of the thrombosis. The prothrombin time was 13.7 seconds and the INR was at 0.98 (normal range: 2-3). Antiphospholipid screening was negative. An ENT examination and referral revealed no localized infection in the ear or mastoid. Thus, a diagnosis of steroid-induced right transverse and sigmoid sinus thrombosis was made in view of no other known causes and a temporal association with dexamethasone pulse therapy. The patient was started on oral anticoagulants and acetazolamide tablets $250 \mathrm{mg}$ twice a day. Oral corticosteroids and azathioprine were stopped. Pulse therapy was withheld. The headache subsided in the next two weeks. To manage the pemphigus, the patient was started on oral mycophenolate mofetil at a dose of $1 \mathrm{~g}$ twice daily with gradual control of disease activity.

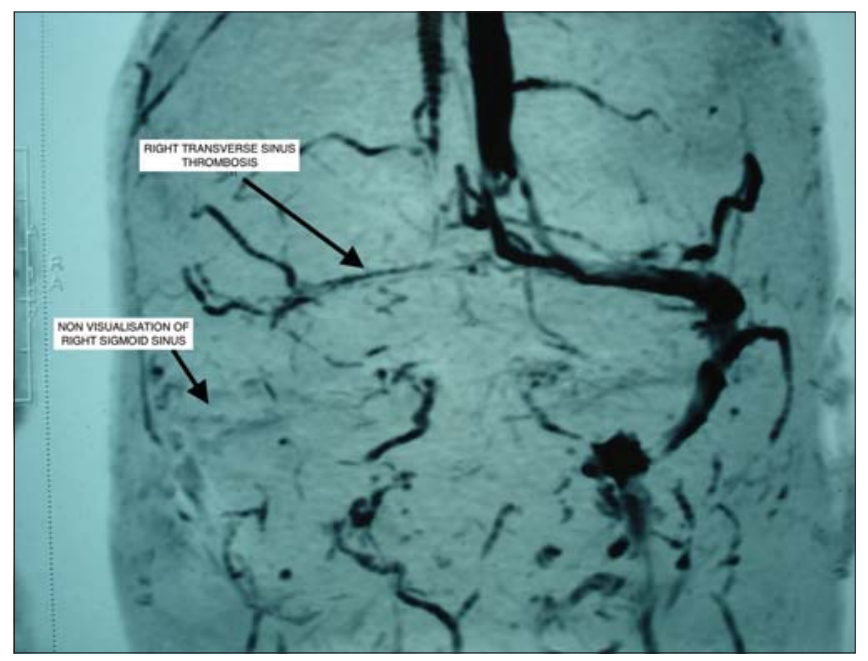

Figure 1: Magnetic resonance venography revealing the attenuation of flow-related enhancement of the right transverse sinus and the most distal part of the superior sagittal sinus with non-visualization of the right sigmoid sinus.

\section{DISCUSSION}

Cerebral venous thrombosis is a rare disease that accounts for $0.5 \%$ of all strokes [5]. It is characterized by clotting of the blood in the cerebral venous or dural sinuses or even the cortical veins [6]. The etiological factors include infections (mastoiditis, otitis, meningitis) and inflammatory diseases. The other predisposing factors are pregnancy, puerperium, trauma, neoplastic disorders, obesity, thrombophilia, contraceptives, and prior neurosurgery [6].

The role of corticosteroids as a procoagulant causing cerebral venous thrombosis has not been elaborately documented in the literature. Cushing's syndrome is a condition involving elevated levels of glucocorticoids. It is also associated with increased von Willebrand factor, a short APTT, the development of thrombin, and the impairment of fibrinolytic activity. All these factors are associated with a procoagulant state [7]. The presence of hypercoagulability causing femoral head necrosis has been reported, even with physiological doses of steroid therapy [8].

\section{CONCLUSION}

The thrombosis in our patient might be attributed to the high dose of corticosteroids in the absence of other risk factors. To our knowledge, there has been no report of cerebral venous thrombosis in a patient with pemphigus vulgaris with the absence of comorbidities and risk factors. However, a case of cerebral venous 
thrombosis due to a high pulse dose of intravenous methylprednisolone in a patient with multiple sclerosis has been reported [9].

\section{Consent}

The examination of the patient was conducted according to the principles of the Declaration of Helsinki.

The authors certify that they have obtained all appropriate patient consent forms, in which the patients gave their consent for images and other clinical information to be included in the journal. The patients understand that their names and initials will not be published and due effort will be made to conceal their identity, but that anonymity cannot be guaranteed.

\section{REFERENCES}

1. Didona D, Maglie R, Eming R, Hertl M. Pemphigus: Current and future therapeutic strategies. Front Immunol. 2019;10:1418.

2. Porro AM, Hans Filho G, Santi CG. Consensus on the treatment of autoimmune bullous dermatoses: Pemphigus vulgaris and pemphigus foliaceus - Brazilian Society of Dermatology. An Bras Dermatol. 2019;94:20-32.
3. Pasricha JS; Poonam. Current regimen of pulse therapy for pemphigus: Minor modifications, improved results. Indian J Dermatol Venereol Leprol. 2008;74:217-21.

4. Abraham A, Roga G, Job AM. Pulse therapy in pemphigus: Ready reckoner. Indian J Dermatol. 2016;61:314-7.

5. Ferro JM, Bousser MG, Canhão P, Coutinho JM, Crassard I, Dentali F, et al. European Stroke Organization guideline for the diagnosis and treatment of cerebral venous thrombosis - Endorsed by the European Academy of Neurology. Eur Stroke J. 2017;2:195-221.

6. Silvis SM, Middeldorp S, Zuurbier SM, Cannegieter SC, Coutinho JM. Risk factors for cerebral venous thrombosis. Semin Thromb Hemost. 2016;42:622-31.

7. van der Pas R, Leebeek FW, Hofland LJ, de Herder WW, Feelders RA. Hypercoagulability in Cushing's syndrome: Prevalence, pathogenesis and treatment. Clin Endocrinol (Oxf). 2013;78:481-8.

8. Rajput S, Kulshreshtha B. Bilateral femoral head avascular necrosis with physiological doses of steroids. Indian J Endocrinol Metab. 2018;22:710-1.

9. Gazioglu S, Solmaz D, Boz C. Cerebral venous thrombosis after high dose steroid in multiple sclerosis: A case report. Hippokratia. 2013;17:88-90.

Copyright by Vijay Gandhi, et al. This is an open access article distributed under the terms of the Creative Commons Attribution License, which permits unrestricted use, distribution, and reproduction in any medium, provided the original author and source are credited.

Source of Support: Nil, Conflict of Interest: None declared. 